\title{
On Strong Convergence for Weighted Sums of a Class of Random Variables
}

\author{
Aiting Shen \\ School of Mathematical Science, Anhui University, Hefei 230601, China \\ Correspondence should be addressed to Aiting Shen; empress201010@126.com
}

Received 5 February 2013; Accepted 27 February 2013

Academic Editor: Simeon Reich

Copyright (C) 2013 Aiting Shen. This is an open access article distributed under the Creative Commons Attribution License, which permits unrestricted use, distribution, and reproduction in any medium, provided the original work is properly cited.

Let $\left\{X_{n}, n \geq 1\right\}$ be a sequence of random variables satisfying the Rosenthal-type maximal inequality. Complete convergence is studied for linear statistics that are weighted sums of identically distributed random variables under a suitable moment condition. As an application, the Marcinkiewicz-Zygmund-type strong law of large numbers is obtained. Our result generalizes the corresponding one of Zhou et al. (2011) and improves the corresponding one of Wang et al. (2011, 2012).

\section{Introduction}

Throughout the paper, let $I(A)$ be the indicator function of the set $A$. $C$ denotes a positive constant which may be different in various places, and $a_{n}=O\left(b_{n}\right)$ stands for $a_{n} \leq$ $C b_{n}$. Denote $\log x=\ln \max (x, e)$.

Let $\left\{X, X_{n}, n \geq 1\right\}$ be a sequence of identically distributed random variables and $\left\{a_{n i}, 1 \leq i \leq n, n \geq 1\right\}$ an array of constants. The strong convergence results for weighted sums $\sum_{i=1}^{n} a_{n i} X_{i}$ have been studied by many authors; see, for example, Choi and Sung [1], Cuzick [2], Wu [3], Bai and Cheng [4], Chen and Gan [5], Cai [6], Sung [7, 8], Shen [9], Wang et al. [10-14], Zhou et al. [15], Wu [16-18], Xu and Tang [19], and so forth. Many useful linear statistics are these weighted sums. Examples include least squares estimators, nonparametric regression function estimators, and jackknife estimates among others. Bai and Cheng [4] proved the strong law of large numbers for weighted sums:

$$
\frac{1}{b_{n}} \sum_{i=1}^{n} a_{n i} X_{i} \longrightarrow 0 \text {, a.s. }
$$

when $\left\{X, X_{n}, n \geq 1\right\}$ is a sequence of independent and identically distributed random variables with $E X=0$ and
$E \exp \left(h|X|^{\gamma}\right)<\infty$ for some $h>0$ and $\gamma>0$, and $\left\{a_{n i}, 1 \leq i \leq\right.$ $n, n \geq 1\}$ is an array of constants satisfying

$$
A_{\alpha} \doteq \limsup _{n \rightarrow \infty} A_{\alpha, n}<\infty, \quad A_{\alpha, n}^{\alpha} \doteq \frac{1}{n} \sum_{i=1}^{n}\left|a_{n i}\right|^{\alpha}
$$

for some $1<\alpha<2$, where $b_{n}=n^{1 / \alpha}(\log n)^{1 / \gamma+\gamma(\alpha-1) / \alpha(1+\gamma)}$.

Cai [6] generalized the result of Bai and Cheng [4] to the case of negatively associated (NA, in short) random variables and obtained the following complete convergence result for weighted sums of identically distributed NA random variables.

Theorem 1. Let $\left\{X, X_{n}, n \geq 1\right\}$ be a sequence of $N A$ random variables with identical distributions. And let $\left\{a_{n i}, 1 \leq i \leq\right.$ $n, n \geq 1\}$ be a triangular array of constants satisfying $\sum_{i=1}^{n}\left|a_{n i}\right|^{\alpha}=O(n)$ for $0<\alpha \leq 2$. Let $b_{n}=n^{1 / \alpha} \log ^{1 / \gamma} n$ for some $\gamma>0$. Furthermore, assume that $E X=0$ when $1<\alpha \leq 2$. If $E \exp \left(h|X|^{\gamma}\right)<\infty$ for some $h>0$, then

$$
\sum_{n=1}^{\infty} \frac{1}{n} P\left(\max _{1 \leq j \leq n}\left|\sum_{i=1}^{j} a_{n i} X_{i}\right|>\varepsilon b_{n}\right)<\infty, \quad \forall \varepsilon>0 .
$$

Recently, Wang et al. [14] extended the result of Cai [6] for sequences of NA random variables to the case of arrays of rowwise negatively orthant-dependent (NOD, in short) random variables. Sung [8] improved the result of Cai [6] 
for NA random variables under much weaker conditions. Zhou et al. [15] generalized the result of Sung [8] to the case of $\rho^{*}$-mixing random variables when $\alpha>\gamma$. The technique used in Sung [8] is the result of Chen et al. [20] for NA random variables, which is not proved for $\rho^{*}$-mixing random variables. The main purpose of the paper is to further study the strong convergence for a class of random variables satisfying the Rosenthal-type maximal inequality by using a different method from that of Sung [8]. We not only generalize the result of Zhou et al. [15] for $\rho^{*}$-mixing random variables to the case of a sequence of random variables satisfying the Rosenthal-type maximal inequality, but also consider the case of $\alpha \leq \gamma$. In addition, our main result improves the corresponding one of Wang et al. [11, 14], since the exponential moment condition is weakened to moment condition.

\section{Main Results}

In this section, we will study the strong convergence for a class of random variables satisfying the Rosenthal-type maximal inequality by using a different method from that of Sung [8]. As an application, the Marcinkiewicz-Zygmund-type strong law of large numbers is obtained.

Theorem 2. Let $\left\{X_{n}, n \geq 1\right\}$ be a sequence of identically distributed random variables. Let $\left\{a_{n i}, 1 \leq i \leq n, n \geq 1\right\}$ be an array of constants satisfying $\sum_{i=1}^{n}\left|a_{n i}\right|^{\alpha}=O(n)$ for some $0<\alpha \leq 2$. $E X_{n}=0$ when $1<\alpha \leq 2$. Let $b_{n}=n^{1 / \alpha} \log ^{1 / \gamma} n$ for some $\gamma>0$. Assume that for any $q \geq 2$, there exists a positive constant $C_{q}$ depending only on $q$ such that

$$
\begin{gathered}
E\left(\max _{1 \leq j \leq n}\left|\sum_{i=1}^{j}\left(Y_{n i}-E Y_{n i}\right)\right|^{q}\right) \\
\leq C_{q}\left[\sum_{i=1}^{n} E\left|Y_{n i}\right|^{q}+\left(\sum_{i=1}^{n} E Y_{n i}^{2}\right)^{q / 2}\right],
\end{gathered}
$$

where $Y_{n i}=-b_{n} I\left(a_{n i} X_{i}<-b_{n}\right)+a_{n i} X_{i} I\left(\left|a_{n i} X_{i}\right| \leq b_{n}\right)+$ $b_{n} I\left(a_{n i} X_{i}>b_{n}\right)$ or $Y_{n i}=a_{n i} X_{i} I\left(\left|a_{n i} X_{i}\right| \leq b_{n}\right)$. Furthermore, suppose that

$$
\sum_{n=1}^{\infty} n^{-1}\left[\sum_{i=1}^{n} P\left(\left|a_{n i} X_{i}\right|>b_{n}\right)\right]^{q / 2}<\infty
$$

for $Y_{n i}=-b_{n} I\left(a_{n i} X_{i}<-b_{n}\right)+a_{n i} X_{i} I\left(\left|a_{n i} X_{i}\right| \leq b_{n}\right)+$ $b_{n} I\left(a_{n i} X_{i}>b_{n}\right)$. If

$$
\begin{gathered}
E\left|X_{1}\right|^{\alpha}<\infty, \quad \text { for } \alpha>\gamma, \\
E\left|X_{1}\right|^{\alpha} \log \left|X_{1}\right|<\infty, \quad \text { for } \alpha=\gamma, \\
E\left|X_{1}\right|^{\gamma}<\infty, \quad \text { for } \alpha<\gamma,
\end{gathered}
$$

then (3) holds.

Proof. We only need to prove that (3) holds for $Y_{n i}=$ $-b_{n} I\left(a_{n i} X_{i}<-b_{n}\right)+a_{n i} X_{i} I\left(\left|a_{n i} X_{i}\right| \leq b_{n}\right)+b_{n} I\left(a_{n i} X_{i}>b_{n}\right)$. The proof for $Y_{n i}=a_{n i} X_{i} I\left(\left|a_{n i} X_{i}\right| \leq b_{n}\right)$ is analogous.
Without loss of generality, we may assume that $\sum_{i=1}^{n}\left|a_{n i}\right|^{\alpha} \leq n$. It is easy to check that for any $\varepsilon>0$,

$$
\begin{aligned}
& \left(\max _{1 \leq j \leq n}\left|\sum_{i=1}^{j} a_{n i} X_{i}\right|>\varepsilon b_{n}\right) \\
& \quad \subset\left(\max _{1 \leq i \leq n}\left|a_{n i} X_{i}\right|>b_{n}\right) \bigcup\left(\max _{1 \leq j \leq n}\left|\sum_{i=1}^{j} Y_{n i}\right|>\varepsilon b_{n}\right),
\end{aligned}
$$

which implies that

$$
\begin{aligned}
& P\left(\max _{1 \leq j \leq n}\left|\sum_{i=1}^{j} a_{n i} X_{i}\right|>\varepsilon b_{n}\right) \\
& \leq P\left(\max _{1 \leq i \leq n}\left|a_{n i} X_{i}\right|>b_{n}\right)+P\left(\max _{1 \leq j \leq n}\left|\sum_{i=1}^{j} Y_{n i}\right|>\varepsilon b_{n}\right) \\
& \leq \sum_{i=1}^{n} P\left(\left|a_{n i} X_{i}\right|>b_{n}\right) \\
&+P\left(\max _{1 \leq j \leq n}\left|\sum_{i=1}^{j}\left(Y_{n i}-E Y_{n i}\right)\right|>\varepsilon b_{n}-\max _{1 \leq j \leq n}\left|\sum_{i=1}^{j} E Y_{n i}\right|\right) .
\end{aligned}
$$

Firstly, we will show that

$$
b_{n}^{-1} \max _{1 \leq j \leq n}\left|\sum_{i=1}^{j} E Y_{n i}\right| \longrightarrow 0, \quad \text { as } n \longrightarrow \infty \text {. }
$$

When $1<\alpha \leq 2$, we have by $E X_{n}=0$, Markov's inequality and (6) that

$$
\begin{aligned}
b_{n}^{-1} \max _{1 \leq j \leq n}\left|\sum_{i=1}^{j} E Y_{n i}\right| \leq & \sum_{i=1}^{n} P\left(\left|a_{n i} X_{i}\right|>b_{n}\right) \\
& +b_{n}^{-1} \max _{1 \leq j \leq n}\left|\sum_{i=1}^{j} a_{n i} E X_{i} I\left(\left|a_{n i} X_{i}\right|>b_{n}\right)\right| \\
\leq & \sum_{i=1}^{n} P\left(\left|a_{n i} X_{1}\right|>b_{n}\right) \\
& +b_{n}^{-1} \sum_{i=1}^{n} E\left|a_{n i} X_{1}\right| I\left(\left|a_{n i} X_{1}\right|>b_{n}\right) \\
\leq & b_{n}^{-\alpha} \sum_{i=1}^{n}\left|a_{n i}\right|^{\alpha} E\left|X_{1}\right|^{\alpha} \\
& +\left.\left.b_{n}^{-\alpha} \sum_{i=1}^{n}\left|a_{n i}\right|^{\alpha} E\right|^{\prime}\right|^{\alpha} \\
\leq & 2 E\left|X_{1}\right|^{\alpha}(\log n)^{-\alpha / \gamma} \longrightarrow 0, \quad \text { as } n \longrightarrow \infty .
\end{aligned}
$$


When $0<\alpha \leq 1$, we have by Markov's inequality and (6) again that

$$
\begin{aligned}
& b_{n}^{-1} \max _{1 \leq j \leq n}\left|\sum_{i=1}^{j} E Y_{n i}\right| \\
& \quad \leq \sum_{i=1}^{n} P\left(\left|a_{n i} X_{i}\right|>b_{n}\right)+b_{n}^{-1} \sum_{i=1}^{n} E\left|a_{n i} X_{i}\right| I\left(\left|a_{n i} X_{i}\right| \leq b_{n}\right) \\
& \quad=\sum_{i=1}^{n} P\left(\left|a_{n i} X_{1}\right|>b_{n}\right)+b_{n}^{-1} \sum_{i=1}^{n} E\left|a_{n i} X_{1}\right| I\left(\left|a_{n i} X_{1}\right| \leq b_{n}\right) \\
& \quad \leq b_{n}^{-\alpha} \sum_{i=1}^{n}\left|a_{n i}\right|^{\alpha} E\left|X_{1}\right|^{\alpha}+b_{n}^{-\alpha} \sum_{i=1}^{n} E\left|a_{n i} X_{1}\right|^{\alpha} I\left(\left|a_{n i} X_{1}\right| \leq b_{n}\right) \\
& \quad \leq b_{n}^{-\alpha} n E\left|X_{1}\right|^{\alpha}+b_{n}^{-\alpha} n E\left|X_{1}\right|^{\alpha} \\
& \quad=2 E\left|X_{1}\right|^{\alpha}(\log n)^{-\alpha / \gamma} \longrightarrow 0, \quad \text { as } n \longrightarrow \infty .
\end{aligned}
$$

By (10) and (11), we can get (9) immediately. Hence, for $n$ large enough,

$$
\begin{aligned}
& P\left(\max _{1 \leq j \leq n}\left|\sum_{i=1}^{j} a_{n i} X_{i}\right|>\varepsilon b_{n}\right) \\
& \quad \leq \sum_{i=1}^{n} P\left(\left|a_{n i} X_{i}\right|>b_{n}\right)+P\left(\max _{1 \leq j \leq n}\left|\sum_{i=1}^{j}\left(Y_{n i}-E Y_{n i}\right)\right|>\frac{\varepsilon}{2} b_{n}\right) .
\end{aligned}
$$

To prove (3), we only need to show that

$$
\begin{gathered}
I \doteq \sum_{n=1}^{\infty} n^{-1} \sum_{i=1}^{n} P\left(\left|a_{n i} X_{i}\right|>b_{n}\right)<\infty \\
J \doteq \sum_{n=1}^{\infty} n^{-1} P\left(\max _{1 \leq j \leq n}\left|\sum_{i=1}^{j}\left(Y_{n i}-E Y_{n i}\right)\right|>\frac{\varepsilon}{2} b_{n}\right)<\infty .
\end{gathered}
$$

Firstly, we will prove (13). By $\sum_{i=1}^{n}\left|a_{n i}\right|^{\alpha} \leq n$ and (6), we can get that

$$
\begin{aligned}
I \leq & \sum_{n=1}^{\infty} n^{-1} b_{n}^{-\alpha} \sum_{i=1}^{n} E\left|a_{n i} X_{1}\right|^{\alpha} I\left(\left|a_{n i} X_{1}\right|>b_{n}\right) \\
\leq & \sum_{n=1}^{\infty} n^{-2}(\log n)^{-\alpha / \gamma} \\
& \times \sum_{i=1}^{n} E\left|a_{n i} X_{1}\right|^{\alpha} I\left(\sum_{i=1}^{n}\left|a_{n i} X_{1}\right|^{\alpha}>n(\log n)^{\alpha / \gamma}\right)
\end{aligned}
$$

$$
\begin{aligned}
& \leq \sum_{n=1}^{\infty} n^{-2}(\log n)^{-\alpha / \gamma} \\
& \times \sum_{i=1}^{n} E\left|a_{n i} X_{1}\right|^{\alpha} I\left(\left|X_{1}\right|^{\alpha}>(\log n)^{\alpha / \gamma}\right) \\
& \leq \sum_{n=1}^{\infty} n^{-1}(\log n)^{-\alpha / \gamma} E\left|X_{1}\right|^{\alpha} I\left(\left|X_{1}\right|^{\gamma}>\log n\right) \\
& =\sum_{n=1}^{\infty} n^{-1}(\log n)^{-\alpha / \gamma} \\
& \times \sum_{m=n}^{\infty} E\left|X_{1}\right|^{\alpha} I\left(\log m<\left|X_{1}\right|^{\gamma} \leq \log (m+1)\right) \\
& =\sum_{m=1}^{\infty} E\left|X_{1}\right|^{\alpha} I\left(\log m<\left|X_{1}\right|^{\gamma} \leq \log (m+1)\right) \\
& \times \sum_{n=1}^{m} n^{-1}(\log n)^{-\alpha / \gamma} \\
& \leq\left\{\begin{array}{c}
C \sum_{m=1}^{\infty} E\left|X_{1}\right|^{\alpha} I\left(\log m<\left|X_{1}\right|^{\gamma} \leq \log (m+1)\right), \\
\text { for } \alpha>\gamma, \\
C \sum_{m=1}^{\infty} E\left|X_{1}\right|^{\alpha} I\left(\log m<\left|X_{1}\right|^{\gamma} \leq \log (m+1)\right) \\
\quad \times \log \log m, \quad \text { for } \alpha=\gamma, \\
C \sum_{m=1}^{\infty} E\left|X_{1}\right|^{\alpha} I\left(\log m<\left|X_{1}\right|^{\gamma} \leq \log (m+1)\right) \\
\times(\log m)^{1-\alpha / \gamma}, \quad \text { for } \alpha<\gamma
\end{array}\right. \\
& \leq \begin{cases}C E\left|X_{1}\right|^{\alpha}, & \text { for } \alpha>\gamma, \\
C E\left|X_{1}\right|^{\alpha} \log \left|X_{1}\right|, & \text { for } \alpha=\gamma, \\
C E\left|X_{1}\right|^{\gamma}, & \text { for } \alpha<\gamma\end{cases} \\
& <\infty \text {, }
\end{aligned}
$$

which implies (13).

In the following, we will prove (14). Let $q>$ $\max \{2, \alpha, \gamma,(2 \gamma / \alpha)\}$. By Markov's inequality and condition (4), we have

$$
\begin{aligned}
J \leq & C \sum_{n=1}^{\infty} n^{-1} b_{n}^{-q} E\left(\max _{1 \leq j \leq n}\left|\sum_{i=1}^{j}\left(Y_{n i}-E Y_{n i}\right)\right|^{q}\right) \\
\leq & C \sum_{n=1}^{\infty} n^{-1} b_{n}^{-q} \sum_{i=1}^{n} E\left|Y_{n i}\right|^{q} \\
& +C \sum_{n=1}^{\infty} n^{-1} b_{n}^{-q}\left(\sum_{i=1}^{n} E Y_{n i}^{2}\right)^{q / 2} \\
\doteq & J_{1}+J_{2} .
\end{aligned}
$$

To prove (14), it suffices to show that $J_{1}<\infty$ and $J_{2}<\infty$. 
For $j \geq 1$ and $n \geq 2$, denote

$$
I_{n j}=\left\{1 \leq i \leq n: \frac{n}{j+1}<\left|a_{n i}\right|^{\alpha} \leq \frac{n}{j}\right\} .
$$

In view of $\sum_{i=1}^{n}\left|a_{n i}\right|^{\alpha} \leq n$, it is easy to see that $\left\{I_{n j}, j \geq 1\right\}$ are disjoint and $\bigcup_{j=1}^{\infty} I_{n j}=\left\{1 \leq i \leq n: a_{n i} \neq 0\right\}$. Hence, we have for all $m \geq 1$ that

$$
\begin{aligned}
n & \geq \sum_{i=1}^{n}\left|a_{n i}\right|^{\alpha}=\sum_{\left\{1 \leq i \leq n: a_{n i} \neq 0\right\}}\left|a_{n i}\right|^{\alpha}=\sum_{j=1}^{\infty} \sum_{i \in I_{n j}}\left|a_{n i}\right|^{\alpha} \\
& \geq n \sum_{j=1}^{\infty}(j+1)^{-1} \sharp I_{n j} \\
& \geq n \sum_{j=m}^{\infty}(j+1)^{-q / \alpha}(j+1)^{q / \alpha-1} \sharp I_{n j} \\
& \geq n \sum_{j=m}^{\infty}(j+1)^{-q / \alpha}(m+1)^{q / \alpha-1} \sharp I_{n j},
\end{aligned}
$$

which implies that for all $m \geq 1$,

$$
\sum_{j=m}^{\infty}(j+1)^{-q / \alpha} \sharp I_{n j} \leq C m^{1-q / \alpha}, \quad n \geq 2 .
$$

By $C_{r}$ 's inequality, (13) and (17), we can get that

$$
\begin{aligned}
J_{1} \leq & C \sum_{n=1}^{\infty} n^{-1} \sum_{i=1}^{n} P\left(\left|a_{n i} X_{i}\right|>b_{n}\right) \\
& +C \sum_{n=1}^{\infty} n^{-1} b_{n}^{-q} \sum_{i=1}^{n} E\left|a_{n i} X_{i}\right|^{q} I\left(\left|a_{n i} X_{i}\right| \leq b_{n}\right) \\
\leq & C \sum_{n=2}^{\infty} n^{-1} b_{n}^{-q} \sum_{i=1}^{n} E\left|a_{n i} X_{1}\right|^{q} I\left(\left|a_{n i} X_{1}\right| \leq b_{n}\right) \\
\leq & C \sum_{n=2}^{\infty} n^{-1-q / \alpha}(\log n)^{-q / \gamma} \\
& \times \sum_{j=1}^{\infty} \sum_{i \in I_{n j}} E\left|a_{n i} X_{1}\right|^{q} I\left(\left|X_{1}\right| \leq(j+1)^{1 / \alpha}(\log n)^{1 / \gamma}\right)
\end{aligned}
$$

$$
\begin{aligned}
\leq & C \sum_{n=2}^{\infty} n^{-1-q / \alpha}(\log n)^{-q / \gamma} \\
& \times \sum_{j=1}^{\infty} n^{q / \alpha} j^{-q / \alpha} E\left|X_{1}\right|^{q} I\left(\left|X_{1}\right| \leq(j+1)^{1 / \alpha}(\log n)^{1 / \gamma}\right) \sharp I_{n j} \\
\leq & C \sum_{n=2}^{\infty} n^{-1}(\log n)^{-q / \gamma} \\
& \times \sum_{j=1}^{\infty} j^{-q / \alpha} E\left|X_{1}\right|^{q} I\left(\left|X_{1}\right| \leq(\log n)^{1 / \gamma}\right) \sharp I_{n j} \\
& +C \sum_{n=2}^{\infty} n^{-1}(\log n)^{-q / \gamma} \\
& \times \sum_{j=1}^{\infty} j^{-q / \alpha} \sum_{k=1}^{j} E\left|X_{1}\right|^{q} I\left(k^{1 / \alpha}(\log n)^{1 / \gamma}<\left|X_{1}\right|\right. \\
& J_{11}+J_{12} .
\end{aligned}
$$

If $\alpha>\gamma$, we have by (19) and $E\left|X_{1}\right|^{\alpha}<\infty$ that

$$
\begin{aligned}
J_{11} & \leq C \sum_{n=2}^{\infty} n^{-1}(\log n)^{-q / \gamma} E\left|X_{1}\right|^{q} I\left(\left|X_{1}\right| \leq(\log n)^{1 / \gamma}\right) \\
& \leq C \sum_{n=2}^{\infty} n^{-1}(\log n)^{-\alpha / \gamma} E\left|X_{1}\right|^{\alpha} I\left(\left|X_{1}\right| \leq(\log n)^{1 / \gamma}\right) \\
& \leq C \sum_{n=2}^{\infty} n^{-1}(\log n)^{-\alpha / \gamma}<\infty .
\end{aligned}
$$

If $\alpha \leq \gamma$, we have by (6) and (19) that

$$
\begin{aligned}
J_{11} \leq & C \sum_{n=2}^{\infty} n^{-1}(\log n)^{-q / \gamma} E\left|X_{1}\right|^{q} I\left(\left|X_{1}\right| \leq(\log n)^{1 / \gamma}\right) \\
\leq & C \sum_{n=2}^{\infty} n^{-1}(\log n)^{-q / \gamma} \\
& \times \sum_{m=2}^{n} E\left|X_{1}\right|^{q} I\left((\log (m-1))^{1 / \gamma}<\left|X_{1}\right| \leq(\log m)^{1 / \gamma}\right) \\
= & C \sum_{m=2}^{\infty} E\left|X_{1}\right|^{q} I\left((\log (m-1))^{1 / \gamma}<\left|X_{1}\right| \leq(\log m)^{1 / \gamma}\right) \\
& \times \sum_{n=m}^{\infty} n^{-1}(\log n)^{-q / \gamma}
\end{aligned}
$$




$$
\begin{aligned}
& \leq C \sum_{m=2}^{\infty}(\log m)^{1-q / \gamma} E\left|X_{1}\right|^{q} I \\
& \quad \times\left((\log (m-1))^{1 / \gamma}<\left|X_{1}\right| \leq(\log m)^{1 / \gamma}\right) \\
& \leq C \sum_{m=2}^{\infty} E\left|X_{1}\right|^{\gamma} I\left((\log (m-1))^{1 / \gamma}<\left|X_{1}\right| \leq(\log m)^{1 / \gamma}\right) \\
& \leq C E\left|X_{1}\right|^{\gamma}<\infty .
\end{aligned}
$$

By (21) and (22), we can get that $J_{11}<\infty$. Next, we will prove that $J_{12}<\infty$.

It follows by (6) and (19) again that

$$
\begin{aligned}
& J_{12}= C \sum_{n=2}^{\infty} n^{-1}(\log n)^{-q / \gamma} \\
& \times \sum_{k=1}^{\infty} E\left|X_{1}\right|^{q} I\left(k^{1 / \alpha}(\log n)^{1 / \gamma}<\left|X_{1}\right|\right. \\
&\left.\leq(k+1)^{1 / \alpha}(\log n)^{1 / \gamma}\right) \\
& \times \sum_{j=k}^{\infty} j^{-q / \alpha} \sharp I_{n j} \\
& \leq C \sum_{n=2}^{\infty} n^{-1}(\log n)^{-q / \gamma} \\
& \times \sum_{k=1}^{\infty} k^{1-q / \alpha} E\left|X_{1}\right|^{q} I \\
& \leq C \sum_{n=2}^{\infty} n^{-1}(\log n)^{-\alpha / \gamma} \\
& \times \sum_{k=1}^{\infty} E\left|X_{1}\right|^{\alpha} I\left(k^{1 / \alpha}(\log n)^{1 / \gamma}<\left|X_{1}\right|\right. \\
&=\left.C \sum_{n=2}^{\infty} n^{-1}(\log n)^{-\alpha / \gamma}(\log n)^{1 / \gamma}<\left|X_{1}\right| \leq(k+1)^{1 / \alpha}(\log n)^{1 / \gamma}\right) \\
& \times \sum_{m=n}^{\infty} E\left|X_{1}\right|^{\alpha} I\left((\log m)^{1 / \gamma}<\left|X_{1}\right| \leq(\log (m+1))^{1 / \gamma}\right) \\
&\left.\leq(k+1)^{1 / \alpha}(\log n)^{1 / \gamma}\right) \\
&
\end{aligned}
$$

$$
\begin{aligned}
& =C \sum_{m=2}^{\infty} E\left|X_{1}\right|^{\alpha} I\left((\log m)^{1 / \gamma}<\left|X_{1}\right| \leq(\log (m+1))^{1 / \gamma}\right) \\
& \times \sum_{n=2}^{m} n^{-1}(\log n)^{-\alpha / \gamma} \\
& \leq\left\{\begin{array}{r}
C \sum_{m=1}^{\infty} E\left|X_{1}\right|^{\alpha} I\left(\log m<\left|X_{1}\right|^{\gamma} \leq \log (m+1)\right), \\
\quad \text { for } \alpha>\gamma, \\
C \sum_{m=1}^{\infty} E\left|X_{1}\right|^{\alpha} I\left(\log m<\left|X_{1}\right|^{\gamma} \leq \log (m+1)\right) \\
\times \log \log m, \quad \text { for } \alpha=\gamma, \\
C \sum_{m=1}^{\infty} E\left|X_{1}\right|^{\alpha} I\left(\log m<\left|X_{1}\right|^{\gamma} \leq \log (m+1)\right) \\
\times(\log m)^{1-\alpha / \gamma}, \quad \text { for } \alpha<\gamma
\end{array}\right. \\
& \leq \begin{cases}C E\left|X_{1}\right|^{\alpha}, & \text { for } \alpha>\gamma, \\
C E\left|X_{1}\right|^{\alpha} \log \left|X_{1}\right|, & \text { for } \alpha=\gamma, \\
C E\left|X_{1}\right|^{\gamma}, & \text { for } \alpha<\gamma\end{cases} \\
& <\infty \text {. }
\end{aligned}
$$

By $J_{11}<\infty$ and $J_{12}<\infty$, we can get that $J_{1}<\infty$.

To prove (14), it suffices to show that $J_{2}<\infty$. By $C_{r}$ 's inequality, conditions (5) and (6), we can get that

$$
\begin{aligned}
J_{2} \leq & C \sum_{n=1}^{\infty} n^{-1}\left[\sum_{i=1}^{n} P\left(\left|a_{n i} X_{i}\right|>b_{n}\right)\right]^{q / 2} \\
& +C \sum_{n=1}^{\infty} n^{-1} b_{n}^{-q}\left[\sum_{i=1}^{n} E\left|a_{n i} X_{i}\right|^{2} I\left(\left|a_{n i} X_{i}\right| \leq b_{n}\right)\right]^{q / 2} \\
\leq & C \sum_{n=1}^{\infty} n^{-1}\left[\sum_{i=1}^{n} b_{n}^{-\alpha} E\left|a_{n i} X_{1}\right|^{\alpha} I\left(\left|a_{n i} X_{1}\right| \leq b_{n}\right)\right]^{q / 2} \\
\leq & C\left(E\left|X_{1}\right|^{\alpha}\right)^{q / 2} \sum_{n=1}^{\infty} n^{-1}(\log n)^{-\alpha q / 2 \gamma}<\infty .
\end{aligned}
$$

Therefore, (14) follows from (16) and $J_{1}<\infty, J_{2}<\infty$ immediately. This completes the proof of the theorem.

The following result provides the MarcinkiewiczZygmund-type strong law of large numbers for weighted sums $\sum_{i=1}^{n} a_{i} X_{i}$ of a class of random variables satisfying the Rosenthal-type maximal inequality.

Theorem 3. Let $\left\{X_{n}, n \geq 1\right\}$ be a sequence of identically distributed random variables. Let $\left\{a_{n}, n \geq 1\right\}$ be a sequence of constants satisfying $\sum_{i=1}^{n}\left|a_{i}\right|^{\alpha}=O(n)$ for some $0<\alpha \leq 2$. $E X_{n}=0$ when $1<\alpha \leq 2$. Let $b_{n}=n^{1 / \alpha} \log ^{1 / \gamma} n$ for some $\gamma>0$. Assume that for any $q \geq 2$, there exists a positive constant $C_{q}$ depending only on $q$ such that (4) holds, where 
$Y_{n i}=-b_{n} I\left(a_{i} X_{i}<-b_{n}\right)+a_{i} X_{i} I\left(\left|a_{i} X_{i}\right| \leq b_{n}\right)+b_{n} I\left(a_{i} X_{i}>b_{n}\right)$ or $Y_{n i}=a_{i} X_{i} I\left(\left|a_{i} X_{i}\right| \leq b_{n}\right)$. Furthermore, suppose that

$$
\sum_{n=1}^{\infty} n^{-1}\left[\sum_{i=1}^{n} P\left(\left|a_{i} X_{i}\right|>b_{n}\right)\right]^{q / 2}<\infty
$$

for $Y_{n i}=-b_{n} I\left(a_{i} X_{i}<-b_{n}\right)+a_{i} X_{i} I\left(\left|a_{i} X_{i}\right| \leq b_{n}\right)+b_{n} I\left(a_{i} X_{i}>\right.$ $\left.b_{n}\right)$. If (6) holds, then

$$
\begin{gathered}
\sum_{n=1}^{\infty} \frac{1}{n} P\left(\max _{1 \leq j \leq n}\left|\sum_{i=1}^{j} a_{i} X_{i}\right|>\varepsilon b_{n}\right)<\infty, \quad \forall \varepsilon>0, \\
\frac{1}{b_{n}} \sum_{i=1}^{n} a_{i} X_{i} \longrightarrow 0 \text { a.s., } \quad \text { as } n \longrightarrow \infty .
\end{gathered}
$$

Proof. The proof of (26) is the same as that of Theorem 2. So the details are omitted. It suffices to show (27). Denote $S_{n}=$ $\sum_{i=1}^{n} a_{i} X_{i}$ for each $n \geq 1$. It follows by (26) that

$$
\begin{aligned}
\infty & >\sum_{n=1}^{\infty} n^{-1} P\left(\max _{1 \leq j \leq n}\left|S_{j}\right|>\varepsilon b_{n}\right) \\
& =\sum_{i=0}^{\infty} \sum_{n=2^{i}}^{2^{i+1}-1} n^{-1} P\left(\max _{1 \leq j \leq n}\left|S_{j}\right|>\varepsilon n^{1 / \alpha}(\log n)^{1 / \gamma}\right) \\
& \geq \frac{1}{2} \sum_{i=1}^{\infty} P\left(\max _{1 \leq j \leq 2^{i}}\left|S_{j}\right|>\varepsilon 2^{(i+1) / \alpha}\left(\log 2^{i+1}\right)^{1 / \gamma}\right) .
\end{aligned}
$$

By Borel-Cantelli lemma, we obtain that

$$
\lim _{i \rightarrow \infty} \frac{\max _{1 \leq j \leq 2^{i}}\left|S_{j}\right|}{2^{(i+1) / \alpha}\left(\log 2^{i+1}\right)^{1 / \gamma}}=0 \text { a.s. }
$$

For all positive integers $n$, there exists a positive integer $i_{0}$ such that $2^{i_{0}-1} \leq n<2^{i_{0}}$. We have by (29) that

$$
\begin{aligned}
\frac{\left|S_{n}\right|}{b_{n}} & \leq \max _{2^{i_{0}-1} \leq n<2^{i_{0}}} \frac{\left|S_{n}\right|}{b_{n}} \\
& \leq \frac{2^{2 / \alpha} \max _{1 \leq j \leq 2^{i_{0}}}\left|S_{j}\right|}{2^{\left(i_{0}+1\right) / \alpha}\left(\log 2^{i_{0}+1}\right)^{1 / \gamma}}\left(\frac{i_{0}+1}{i_{0}-1}\right)^{1 / \gamma} \longrightarrow 0 \text { a.s., }
\end{aligned}
$$

$$
\text { as } i_{0} \longrightarrow \infty \text {, }
$$

which implies (27). This completes the proof of the theorem.

If the Rosenthal type inequality for the maximal partial sum is replaced by the partial sum, then we can get the following complete convergence result for a class of random variables. The proof is similar to that of Theorem 2. So the details are omitted.

Theorem 4. Let $\left\{X_{n}, n \geq 1\right\}$ be a sequence of identically distributed random variables. Let $\left\{a_{n i}, 1 \leq i \leq n, n \geq 1\right\}$ be an array of constants satisfying $\sum_{i=1}^{n}\left|a_{n i}\right|^{\alpha}=O(n)$ for some $0<\alpha \leq 2$. $E X_{n}=0$ when $1<\alpha \leq 2$. Let $b_{n}=n^{1 / \alpha} \log ^{1 / \gamma} n$ for some $\gamma>0$. Assume that for any $q \geq 2$, there exists a positive constant $C_{q}$ depending only on $q$ such that

$$
\begin{gathered}
E\left(\left|\sum_{i=1}^{n}\left(Y_{n i}-E Y_{n i}\right)\right|^{q}\right) \\
\leq C_{q}\left[\sum_{i=1}^{n} E\left|Y_{n i}\right|^{q}+\left(\sum_{i=1}^{n} E Y_{n i}^{2}\right)^{q / 2}\right],
\end{gathered}
$$

where $Y_{n i}=-b_{n} I\left(a_{n i} X_{i}<-b_{n}\right)+a_{n i} X_{i} I\left(\left|a_{n i} X_{i}\right| \leq b_{n}\right)+$ $b_{n} I\left(a_{n i} X_{i}>b_{n}\right)$ or $Y_{n i}=a_{n i} X_{i} I\left(\left|a_{n i} X_{i}\right| \leq b_{n}\right)$. Furthermore, suppose that (5) holds for $Y_{n i}=-b_{n} I\left(a_{n i} X_{i}<-b_{n}\right)+$ $a_{n i} X_{i} I\left(\left|a_{n i} X_{i}\right| \leq b_{n}\right)+b_{n} I\left(a_{n i} X_{i}>b_{n}\right)$. If (6) satisfies, then

$$
\sum_{n=1}^{\infty} \frac{1}{n} P\left(\left|\sum_{i=1}^{n} a_{n i} X_{i}\right|>\varepsilon b_{n}\right)<\infty, \quad \forall \varepsilon>0 .
$$

Remark 5. There are many sequences of dependent random variables satisfying (4) for all $q \geq 2$. Examples include sequences of NA random variables (see Shao [21]), $\rho^{*}$-mixing random variables (see Utev and Peligrad [22]), $\varphi$-mixing random variables with the mixing coefficients satisfying certain conditions (see Wang et al. [23]), $\rho^{-}$-mixing random variables with the mixing coefficients satisfying certain conditions (see Wang and Lu [24]), and asymptotically almost negatively associated random variables (see Yuan and An [25]). There are also many sequences of dependent random variables satisfying (31) for all $q \geq 2$. Examples not only include the sequences of above, but also include sequences of NOD random variables (see Asadian et al. [26]) and extended negatively dependent random variables (see Shen [27]).

\section{Acknowledgments}

The authors are most grateful to the Editor Simeon Reich and an anonymous referee for careful reading of the manuscript and valuable suggestions which helped in improving an earlier version of this paper. This work is supported by the National Natural Science Foundation of China (11201001, 11171001, and 11126176), the Specialized Research Fund for the Doctoral Program of Higher Education of China (20093401120001), the Natural Science Foundation of Anhui Province (1308085QA03, 11040606M12, 1208085QA03), the 211 project of Anhui University, the Youth Science Research Fund of Anhui University, and the Students Science Research Training Program of Anhui University (KYXL2012007).

\section{References}

[1] B. D. Choi and S. H. Sung, "Almost sure convergence theorems of weighted sums of random variables," Stochastic Analysis and Applications, vol. 5, no. 4, pp. 365-377, 1987.

[2] J. Cuzick, "A strong law for weighted sums of i.i.d. random variables," Journal of Theoretical Probability, vol. 8, no. 3, pp. 625-641, 1995.

[3] W. B. Wu, "On the strong convergence of a weighted sum," Statistics \& Probability Letters, vol. 44, no. 1, pp. 19-22, 1999. 
[4] Z. D. Bai and P. E. Cheng, "Marcinkiewicz strong laws for linear statistics," Statistics \& Probability Letters, vol. 46, no. 2, pp. 105$112,2000$.

[5] P. Chen and S. Gan, "Limiting behavior of weighted sums of i.i.d. random variables," Statistics \& Probability Letters, vol. 77, no. 16, pp. 1589-1599, 2007.

[6] G.-h. Cai, "Strong laws for weighted sums of NA random variables," Metrika, vol. 68, no. 3, pp. 323-331, 2008.

[7] S. H. Sung, "Strong laws for weighted sums of i.i.d. random variables," Statistics \& Probability Letters, vol. 52, no. 4, pp. 413419, 2001.

[8] S. H. Sung, "On the strong convergence for weighted sums of random variables," Statistical Papers, vol. 52, no. 2, pp. 447-454, 2011.

[9] A. Shen, "Some strong limit theorems for arrays of rowwise negatively orthant-dependent random variables," Journal of Inequalities and Applications, vol. 2011, article 93, 2011.

[10] X. Wang, S. Hu, and A. I. Volodin, "Strong limit theorems for weighted sums of NOD sequence and exponential inequalities," Bulletin of the Korean Mathematical Society, vol. 48, no. 5, pp. 923-938, 2011.

[11] X. Wang, S. Hu, and W. Yang, "Complete convergence for arrays of rowwise asymptotically almost negatively associated random variables," Discrete Dynamics in Nature and Society, vol. 2011, Article ID 717126, 11 pages, 2011.

[12] X. Wang, S. Hu, W. Yang, and X. Wang, "On complete convergence of weighted sums for arrays of rowwise asymptotically almost negatively associated random variables," Abstract and Applied Analysis, vol. 2012, Article ID 315138, 15 pages, 2012.

[13] X. Wang, X. Li, W. Yang, and S. Hu, "On complete convergence for arrays of rowwise weakly dependent random variables," Applied Mathematics Letters, vol. 25, no. 11, pp. 1916-1920, 2012.

[14] X. Wang, S. Hu, and W. Yang, "Complete convergence for arrays of rowwise negatively orthant dependent random variables," Revista de la Real Academia de Ciencias Exactas, Físicas y Naturales, vol. 106, no. 2, pp. 235-245, 2012.

[15] X.-C. Zhou, C.-C. Tan, and J.-G. Lin, "On the strong laws for weighted sums of $\rho^{*}$-mixing random variables," Journal of Inequalities and Applications, vol. 2011, Article ID 157816, 8 pages, 2011.

[16] Q. Wu, "A strong limit theorem for weighted sums of sequences of negatively dependent random variables," Journal of Inequalities and Applications, vol. 2010, Article ID 383805, 8 pages, 2010.

[17] Q. Wu, "Sufficient and necessary conditions of complete convergence for weighted sums of PNQD random variables," Journal of Applied Mathematics, vol. 2012, Article ID 104390, 10 pages, 2012.

[18] Q. Wu, "A complete convergence theorem for weighted sums of arrays of rowwise negatively dependent random variables," Journal of Inequalities and Applications, vol. 2012, article 50, 2012.

[19] H. Xu and L. Tang, "Some convergence properties for weighted sums of pairwise NQD sequences," Journal of Inequalities and Applications, vol. 2012, article 255, 2012.

[20] P. Chen, T.-C. Hu, X. Liu, and A. Volodin, "On complete convergence for arrays of rowwise negatively associated random variables," Theory of Probability and its Applications, vol. 52, no. 2, pp. 393-397, 2007.

[21] Q.-M. Shao, "A comparison theorem on moment inequalities between negatively associated and independent random variables," Journal of Theoretical Probability, vol. 13, no. 2, pp. 343$356,2000$.
[22] S. Utev and M. Peligrad, "Maximal inequalities and an invariance principle for a class of weakly dependent random variables," Journal of Theoretical Probability, vol. 16, no. 1, pp. 101$115,2003$.

[23] X. Wang, S. Hu, W. Yang, and Y. Shen, "On complete convergence for weighed sums of $\varphi$-mixing random variables," Journal of Inequalities and Applications, vol. 2010, Article ID 372390, 13 pages, 2010.

[24] J. F. Wang and F. B. Lu, "Inequalities of maximum of partial sums and weak convergence for a class of weak dependent random variables," Acta Mathematica Sinica (English Series), vol. 22, no. 3, pp. 693-700, 2006.

[25] D. Yuan and J. An, "Rosenthal type inequalities for asymptotically almost negatively associated random variables and applications," Science in China A, vol. 52, no. 9, pp. 1887-1904, 2009.

[26] N. Asadian, V. Fakoor, and A. Bozorgnia, "Rosenthal's type inequalities for negatively orthant dependent random variables," Journal of the Iranian Statistical Society, vol. 5, no. 1-2, pp. 66-75, 2006.

[27] A. Shen, "Probability inequalities for END sequence and their applications," Journal of Inequalities and Applications, vol. 2011, article 98, 2011. 


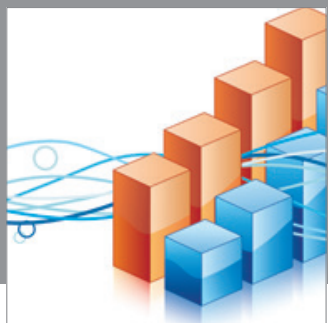

Advances in

Operations Research

mansans

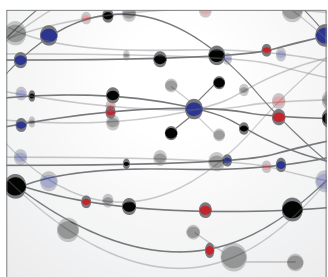

The Scientific World Journal
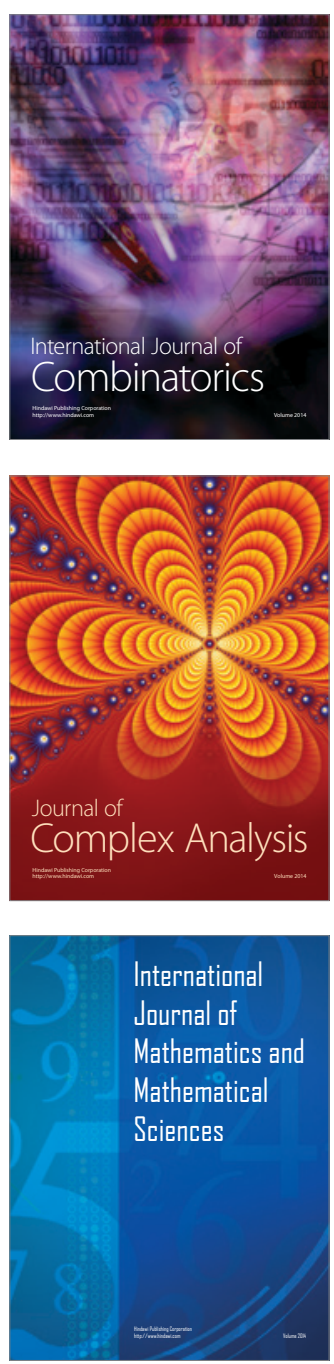
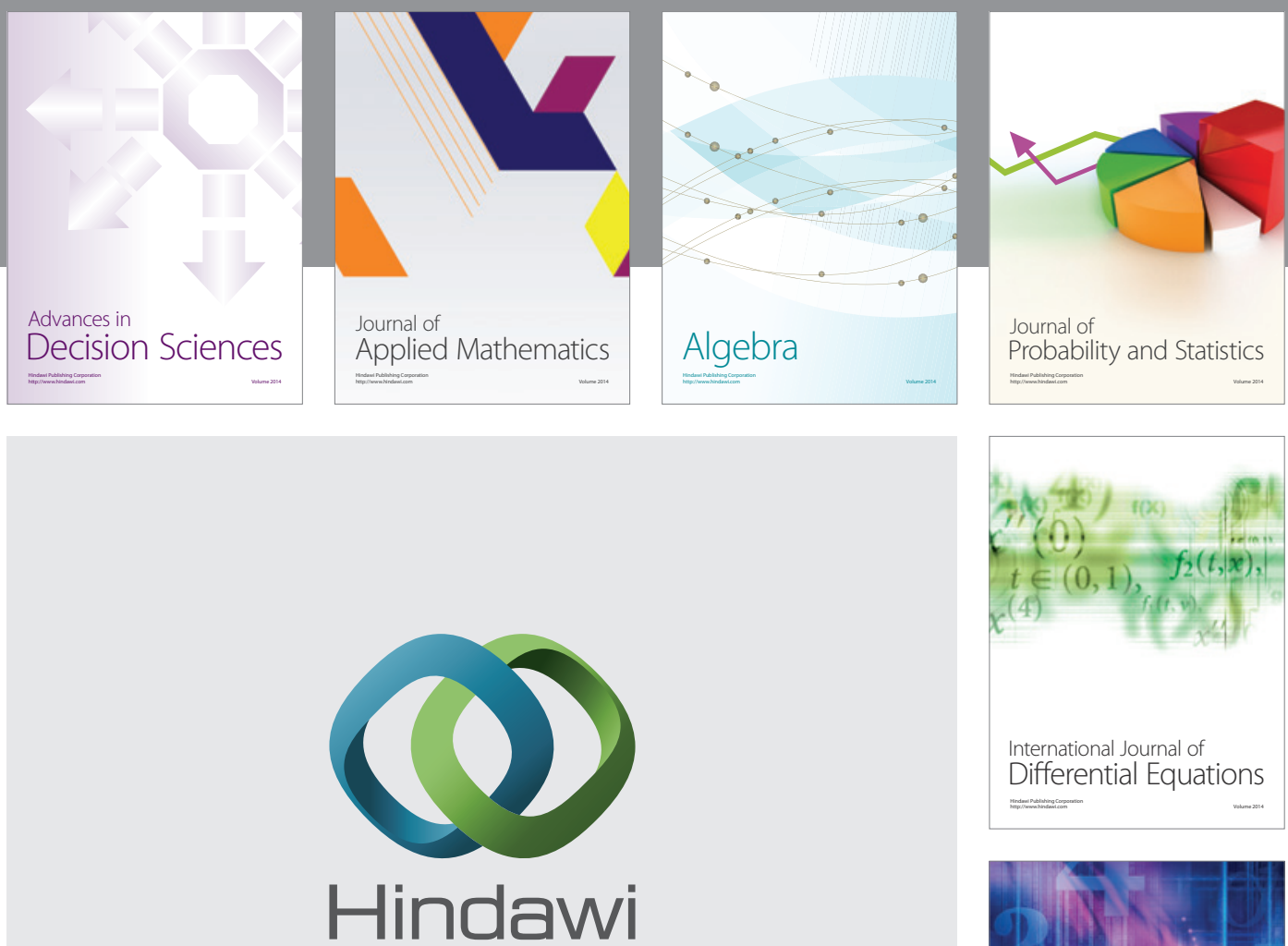

Submit your manuscripts at http://www.hindawi.com
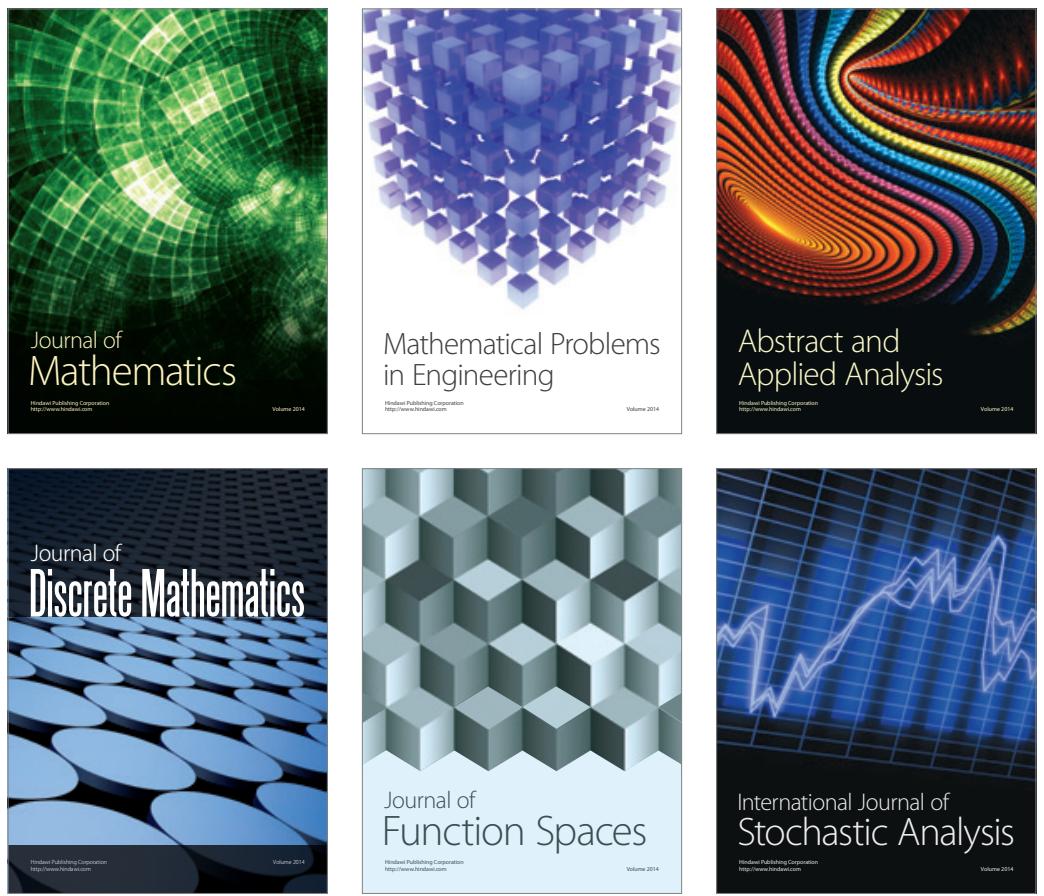

Journal of

Function Spaces

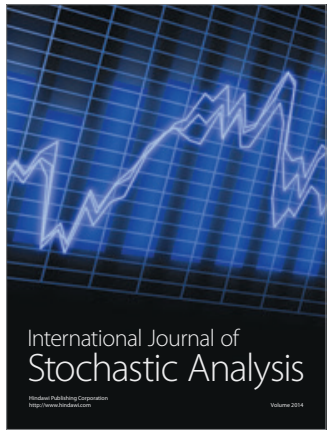

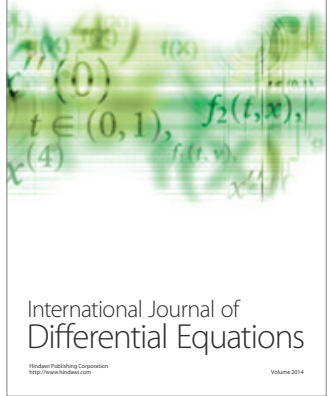
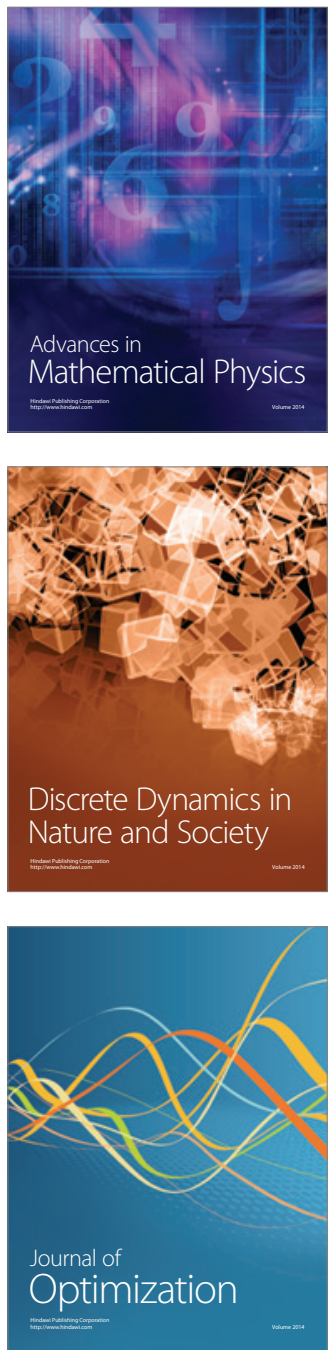